\title{
The K20 survey. V. The evolution of the near-IR Luminosity Function ${ }^{\star}$
}

\author{
L. Pozzetti ${ }^{1}$, A. Cimatti ${ }^{2}$, G. Zamorani ${ }^{1}$, E. Daddi ${ }^{3}$, N. Menci ${ }^{4}$, A. Fontana ${ }^{4}$, A. Renzini ${ }^{3}$, M. Mignoli ${ }^{1}$, F. Poli ${ }^{5}$, \\ P. Saracco ${ }^{6}$, T. Broadhurst ${ }^{3,7}$, S. Cristiani ${ }^{8,9}$, S. D’Odorico ${ }^{3}$, E. Giallongo ${ }^{4}$, and R. Gilmozzi ${ }^{3}$
}

${ }^{1}$ Istituto Nazionale di Astrofisica, Osservatorio Astronomico di Bologna, Via Ranzani 1, 40127 Bologna, Italy

2 Istituto Nazionale di Astrofisica, Osservatorio Astrofisico di Arcetri, Largo E. Fermi 5, 50125 Firenze, Italy

3 European Southern Observatory, Karl-Schwarzschild-Str. 2, 85748, Garching, Germany

${ }^{4}$ Istituto Nazionale di Astrofisica, Osservatorio Astronomico di Roma, via Dell'Osservatorio 2, Monteporzio, Italy

5 Dipartimento di Astronomia, Università "La Sapienza", Roma, Italy

${ }^{6}$ Istituto Nazionale di Astrofisica, Osservatorio Astronomico di Brera, via E. Bianchi 46, Merate, Italy

7 Racah Institute for Physics, The Hebrew University, Jerusalem, 91904, Israel

8 ST, European Coordinating Facility, Karl-Schwarzschild-Str. 2, 85748, Garching, Germany

9 Istituto Nazionale di Astrofisica, Osservatorio Astronomico di Trieste, via Tiepolo 11, Trieste, Italy

Received 28 October 2002 / Accepted 21 February 2003

\begin{abstract}
We present the galaxy rest-frame near-IR Luminosity Function (LF) and its cosmic evolution to $z \sim 1.5$ based on a spectroscopic survey of a magnitude limited sample of galaxies with $K_{\mathrm{s}}<20$ (the K20 survey, Cimatti et al. 2002b). The LFs have been derived in the rest-frame $J$ and $K_{\mathrm{s}}$ bands. Their evolution is traced using three different redshift bins $\left(z_{\text {mean }} \simeq\right.$ $0.5,1,1.5)$ and comparing them to the Local near-IR Luminosity Function. The luminosity functions at different redshifts are fairly well fitted by Schechter functions at $z<1.3$. The faint-end of the LFs $\left(L<L^{*}\right)$ is consistent with the local estimates, with no evidence for a change either in the slope or normalization up to $z<1.3$. At higher redshift this part of the luminosity function is not well sampled by our data. Viceversa, the density of luminous galaxies $\left(M_{K_{\mathrm{s}}}-5 \log h_{70}<-25.5\right)$ is higher than locally at all redshifts and relatively constant or mildly increasing with redshift within our sample. The data are consistent with a mild luminosity evolution both in the $J$ - and $K_{\mathrm{s}}$-band up to $z \simeq 1.5$, with an amplitude of about $\Delta M_{J} \simeq-0.69 \pm 0.12$ and $\Delta M_{K} \simeq-0.54 \pm 0.12$ at $z \sim 1$. Pure density evolution is not consistent with the observed LF at $z \leq 1$. Moreover, we find that red and early-type galaxies dominate the bright-end of the $L F$, and that their number density shows at most a small decrease $(<30 \%)$ up to $z \simeq 1$, thus suggesting that massive elliptical galaxies were already in place at $z \simeq 1$ and they should have formed their stars and assembled their mass at higher redshift. There appears to be a correlation of the optical/near-IR colors with near-IR luminosities, the most luminous/massive galaxies being red/old, the low-luminous galaxies being instead dominated by blue young stellar populations. We also investigate the evolution of the near-IR comoving luminosity density to $z \simeq 1.5$, finding a slow evolution with redshift $\left(\rho_{\lambda}(z)=\rho_{\lambda}(z=0)(1+z)^{\beta(\lambda)}\right.$ with $\beta(J) \simeq 0.70$ and $\left.\beta\left(K_{\mathrm{s}}\right) \simeq 0.37\right)$. Finally, we compare the observed LFs with the predictions of a set of the most updated hierarchical merging models. Such a comparison shows that the current versions of hierarchical models overpredict significantly the density of low luminosity galaxies at $z \leq 1$ and underpredict the density of luminous galaxies at $z \geq 1$, whereas passive evolution models are more consistent with the data up to $z \sim 1.5$. The GIF model (Kaufmann et al. 1999) shows a clear deficiency of red luminous galaxies at $z \sim 1$ compared to our observations and predicts a decrease of luminous galaxies with redshift not observed in our sample.
\end{abstract}

Key words. galaxies: elliptical and lenticular, evolution, formation, luminosity function - cosmology: observations infrared: galaxies

\section{Introduction}

Over the past few years, a wealth of observations from deep surveys of optically-selected high-redshift galaxies (e.g. Madau et al. 1996; Steidel et al. 1999), complemented by observations in the far-IR/sub-mm (Hughes et al. 1998;

\footnotetext{
Send offprint requests to: L. Pozzetti, e-mail: lucia@bo. astro.it

* Based on observations made at the European Southern Observatory, Chile (ESO LP 164.O-0560).
}

Barger et al. 1999), allowed significant progress in our understanding of the evolution of galaxies from the present-epoch back to $z \simeq 4$ and beyond (Steidel et al. 1999; Madau et al. 1998). However, since these samples of high- $z$ galaxies were all selected at optical or far-IR/sub-mm wavelengths, they are dominated by objects with on-going star formation. Therefore, such studies placed constraints more on the evolution of the star birth rate activity than on the formation and assembly of stellar systems through cosmic time. 
The study of faint galaxy samples selected in the nearinfrared represents an important and complementary possibility to address the still open questions on how the formation and evolution of massive systems evolved with time compared to the predictions of the different theoretical scenarios (Broadhurst et al. 1992). Another advantage of the near-IR selection (in particular in the $K$-band) is that the $k$-corrections are relatively insensitive to galaxy type and fairly small also at high redshift (Cowie et al. 1994), and the dust extinction effects are less severe than in optical samples.

Since the rest-frame near-IR light is a relatively good tracer of the galaxy stellar mass (Gavazzi et al. 1996; Madau et al. 1998) the near-IR galaxy Luminosity Function (LF) can provide a reasonable estimate of the Galaxy Stellar Mass Function (GSMF). Only recently the 2MASS (Jarrett et al. 2000) surveys allowed accurate determinations of the local near-IR luminosity and of the Galaxy Stellar-mass functions (Cole et al. 2001; Kochanek et al. 2001), while only few attempts have been made to reconstruct their evolution with redshift using deep surveys of near-IR selected samples (i.e. Cowie et al. 1996; Cohen et al. 1999; Cohen 2002).

In order to address the above questions, we performed a new spectroscopic survey of a complete sample of galaxies selected with $K_{\mathrm{s}}<20$ (the K20 survey; http://www.arcetri.astro.it/ k20/). The survey and the sample are described in detail in Cimatti et al. (2002b, hereafter Paper III), while the spectral and clustering properties of the Extremely Red Objects (EROs) are discussed in Cimatti et al. (2002a, Paper I) and Daddi et al. (2002, Paper II), respectively. The redshift distribution for the whole sample of $K_{\mathrm{s}}$-band selected galaxies is given in Cimatti et al. (2002c, Paper IV). Here we recall that the K20 sample includes 546 objects to $K_{\mathrm{s}}<20$ (Vega system), selected from a $32.2 \operatorname{arcmin}^{2}$ area of the Chandra Deep Field South (CDFS; Giacconi et al. 2001) and from a $19.8 \mathrm{arcmin}^{2}$ field centered at 0055-269. The total area of the two fields is $52 \mathrm{arcmin}^{2}$. Optical multi-object spectroscopy was mainly obtained with the ESO VLT + FORS1 and FORS2, while a small fraction $(\sim 4 \%)$ of the objects was observed with near-IR spectroscopy using the VLT + ISAAC. We have imaged the 0055269 field over 10 bands $\left(U B G V R R_{w} I z J K_{\mathrm{s}}\right)$, obtained with the ESO NTT + SUSI2 $\left(U B G V R_{w} I\right)$ and SOFI $\left(J K_{\mathrm{s}}\right)$, and VLT + FORS1 ( $R$ and $z$ ), for a total of about 45 hours of integration. In the CDFS field, we used a combination of public EIS NTT data $\left(U K_{\mathrm{s}}\right)$ and deep FORS1 images (BVRIz, courtesy of P. Rosati \& M. Nonino). The spectroscopic redshift completeness is $94 \%$ and $87 \%$ for $K_{\mathrm{s}}<19$ and $K_{\mathrm{s}}<20$, respectively, and it increases to $98 \%$ if we include the photometric redshifts obtained with the deep multi-band imaging for the spectroscopically unidentified or unobserved objects (Cimatti et al. 2002b). The K20 sample is the largest and most complete spectroscopic sample of galaxies with $K_{\mathrm{s}}<20$ available to date.

In this paper, we investigate the evolution of the nearIR luminosity function up to $z \simeq 1.5$ based on the K20 survey sample. Thanks to the higher statistical significance and completeness of our sample, it is possible to use the LF evolution to place new and more stringent constraints on the formation and evolution of massive galaxies than was

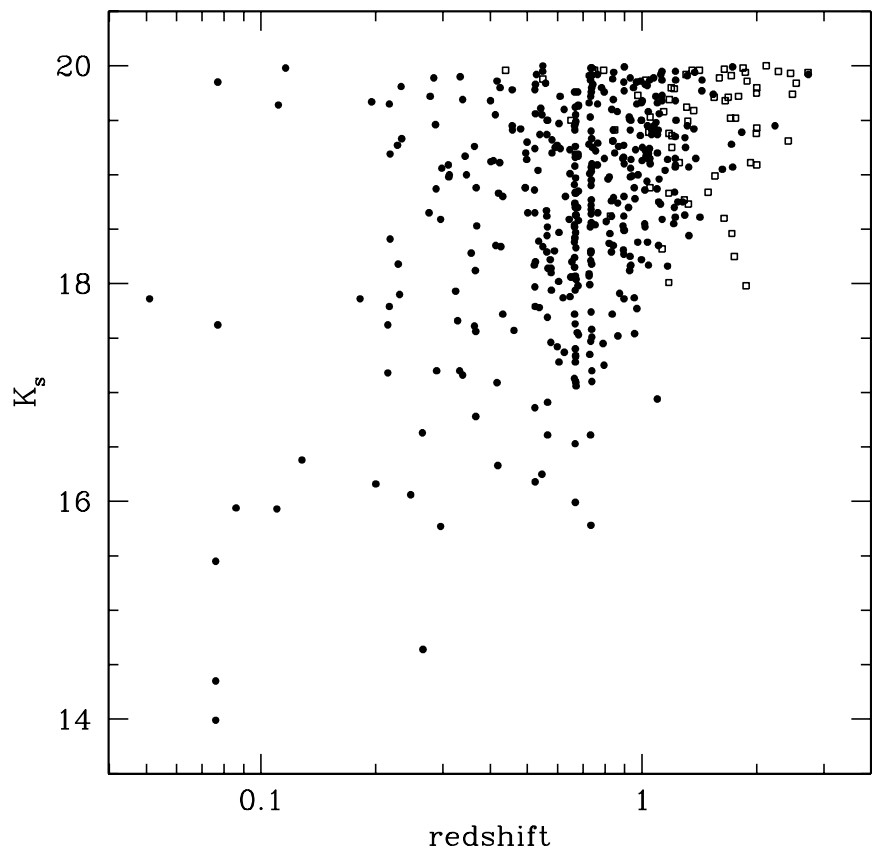

Fig. 1. The magnitude-redshift diagram for all the galaxies in the spectroscopic sample to $K_{\mathrm{s}}<20$. Filled circles represent galaxies identified spectroscopically, while empty squares are unidentified or unobserved galaxies plotted at $z=z_{\text {phot }}$.

possible from previous surveys (Cowie et al. 1996; Cohen et al. 1999; Cohen 2002). In particular our survey has the advantage to include a complete sample of EROs, partially with spectroscopic identifications, which usually were not included in previous spectroscopic surveys (e.g. Cohen 2002) because of their selection. Finally, we compare the LF evolution with the predictions of two competitive scenarios of galaxy evolution: the Pure Luminosity Evolution (PLE) and the Hierarchical Merging Models (HMM). We adopt $H_{0}=70 \mathrm{~km} \mathrm{~s}^{-1} \mathrm{Mpc}^{-1}$, $\Omega_{m}=0.3, \Omega_{\Lambda}=0.7$.

\section{The K20 spectroscopic sample}

From the total K20 sample of 546 objects with $K_{\mathrm{s}} \leq 20$ we have extracted a sample of 489 galaxies, after excluding objects classified as stars and AGN on the basis of their spectra. In addition to spectroscopic redshifts, photometric redshifts have been derived (Cimatti et al. 2002b) with very high accuracy $\left(\left\langle z_{\text {spe }}-z_{\text {phot }}\right\rangle=\langle\Delta z\rangle=0.012\right.$ and $\left.\sigma_{\Delta z}=0.089\left(1+z_{\text {spe }}\right)\right)$. Such an accuracy, made possible by the numerous photometric bands and the precision of the magnitude measurements, is consistent with previous results on the HDF-N data set (Fontana et al. 2000) and with simulations made by Bolzonella et al. (2000) at high redshift $(1<z<2)$, where our sample is the largest to date. The magnitude-redshift distribution of selected galaxies is shown in Fig. 1. Thanks to the depth and completeness of our sample, the coverage of the $K_{\mathrm{s}}$-redshift plane is such that the near-IR LF can be derived with good accuracy and over a relatively large range of magnitudes at least up to $z \sim 1$, still sampling with relatively good statistics the high luminosity part of the LF up to $z \sim 2$. Since the sample spans a wide range in luminosity, redshift and look-back time, it is therefore 


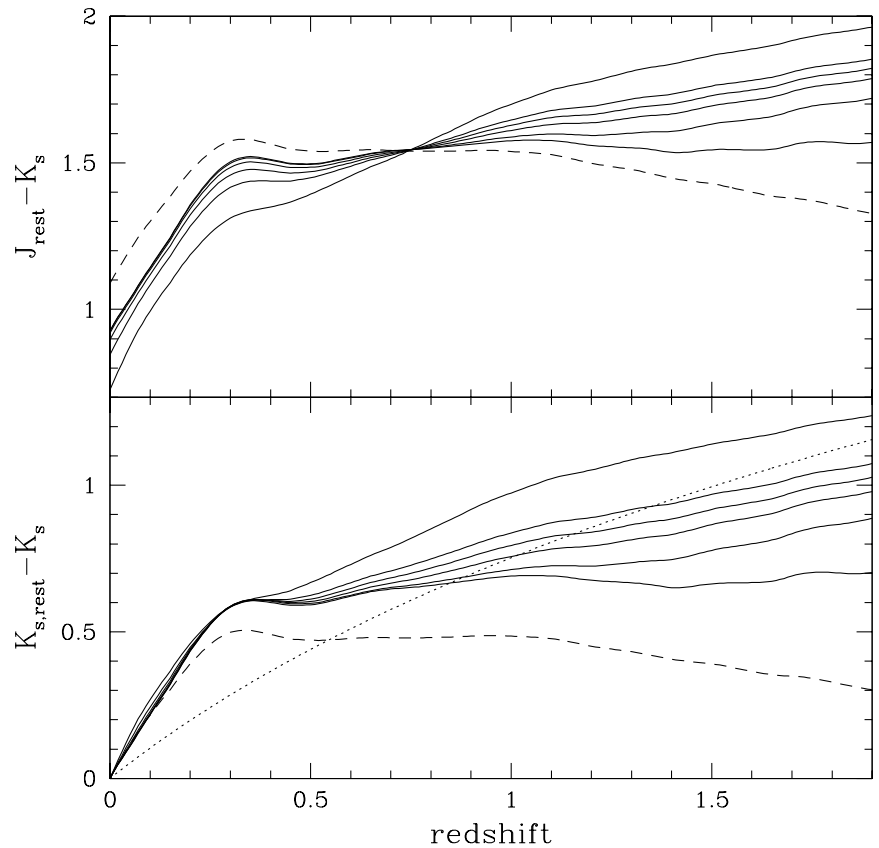

Fig. 2. $k$-corrections colors (see text) as a function of redshift: $J_{\text {rest }}-K_{\mathrm{s}}$ (top panel) and $K_{\mathrm{s}, \text { rest }}-K_{\mathrm{s}}$ (bottom panel). Different curves are derived from different spectral models which at low redshift reproduce spectra and colors of local E, S0, Sa, Sb, Sc, Im (solid lines from bottom to top at $z>1$ ) and dusty star-forming galaxies (dashed line; see text). Dotted line shows the $2.5 \log (1+z)$ term for comparison.

well suited to study the evolution of the near-IR Luminosity Function within the sample itself and in comparison to the local population.

\section{The estimate of the rest-frame near-IR luminosities}

The small variance of $k$-corrections in the $K_{\mathrm{s}}$-band (Cowie et al. 1994; Mannucci et al. 2001) greatly simplifies the analysis with respect to optically-selected samples of galaxies. Moreover, we take advantage of the fact that at $z \simeq 0.8$ the observed $K_{\mathrm{s}}$-band corresponds to rest-frame $J$-band. In order to compute the near-IR Luminosity Function, we derive the rest-frame $J$ and $K_{\mathrm{s}}$-band absolute magnitudes $\left(M_{J}\right.$ and $\left.M_{K_{\mathrm{s}}}\right)$ according to the following relations:

$$
\begin{aligned}
& M_{J}=K_{\mathrm{s}}-5 \log \left(d_{\mathrm{L}}(z) / 10 \mathrm{pc}\right)+\left(J_{\text {rest }}-K_{\mathrm{s}}\right)(z) \\
& M_{K_{\mathrm{s}}}=K_{\mathrm{s}}-5 \log \left(d_{\mathrm{L}}(z) / 10 \mathrm{pc}\right)+\left(K_{\mathrm{s}, \text { rest }}-K_{\mathrm{s}}\right)(z),
\end{aligned}
$$

where $K_{\mathrm{s}}$ is the total apparent magnitude measured with SExtractor (see Cimatti et al. 2002b, Paper III, for more details), $d_{\mathrm{L}}(z)$ is the luminosity distance at redshift $z$ and $\left(J_{\text {rest }}-\right.$ $\left.K_{\mathrm{s}}\right)$ and $\left(K_{\mathrm{s} \text {, rest }}-K_{\mathrm{s}}\right)$ are the " $k$-correction colors", i.e. the difference between rest frame and observed magnitude which includes also the $2.5 \log (1+z)$ term (see also Lilly et al. 1995). It should be noted that in Eq. (1) the last term can be written as a conventional $k$-correction for the $K_{\mathrm{s}}$-band (as in Eq. (2)) plus a rest-frame $\left(J-K_{\mathrm{s}}\right)$ color. The $k$-correction colors as a function of redshift are plotted in Fig. 2. This figure clearly shows the small variation of the $k$-corrections for different spectral types

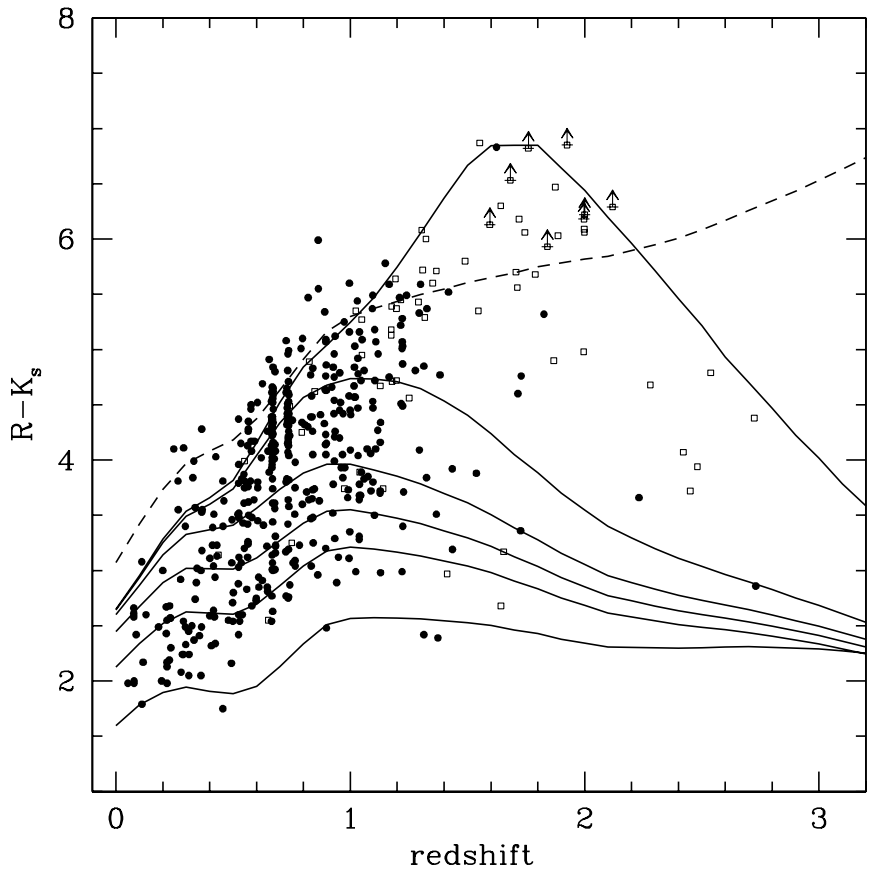

Fig. 3. The color-redshift ( $R-K_{\mathrm{s}}$ vs. $z$ ) diagram for galaxies in the spectroscopic sample to $K_{\mathrm{s}}<20$. Different symbols as in Fig. 1 . Arrows indicate $3 \sigma$ lower limits in $R-K_{\mathrm{s}}$ colors for objects not detected in the $R$ band. The curves show different spectral models as in Fig. 2.

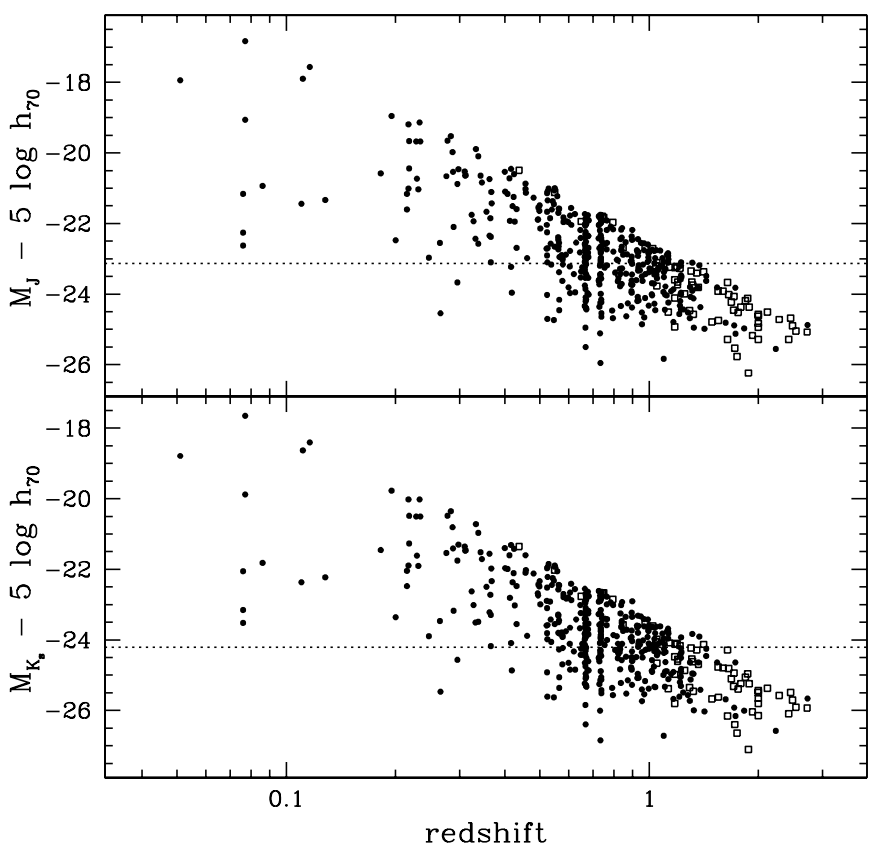

Fig. 4. The absolute $J$ and $K_{\mathrm{s}}$ magnitudes as a function of redshift derived (see text) for all galaxies in the spectroscopic sample to $K_{\mathrm{s}}<20$. Symbols as in Fig. 1. Local estimates of $M_{J}^{*}$ and $M_{K_{\mathrm{s}}}^{*}$ (dotted horizontal lines) from Cole et al. (2001).

over most of the redshift range covered by our data (for a comparison with a similar plot in the optical bands see Lilly et al. 1995).

These $k$-corrections for different spectral types are computed using the Bruzual \& Charlot (1993) models 
(GISSEL 2000 version), in order to reproduce spectra, and $k$-corrections of local galaxies (E, S0, Sa, Sb, Sc, Im) at low redshift (cf. Pozzetti et al. 1996; Mannucci et al. 2001) and the color-redshift diagram $\left(R-K_{\mathrm{s}}\right.$ vs. $\left.z\right)$ observed in our sample (Fig. 3). In order to take into account the dusty star-forming population, we introduce an additional spectral type dominated by star formation and strong dust extinction consistently describing the SED of red emission line objects and reproducing the sub-class of emission line EROs with $R-K_{\mathrm{s}}>5$ (cf. Paper I). We have adopted an exponentially declining star formation history $(S F R \propto \exp (-t / \tau))$, with $\tau=0.3,1,2,4, \infty$ Gyrs (E, S0, Sa, Sb, Sc galaxies, respectively), the Salpeter (1955) Initial Mass Function (IMF) and an age of 12.5 Gyrs at $z=0$ (i.e. a formation redshift $z_{\mathrm{f}}=5.7$ ). A model with an age of $1 \mathrm{Gyr}$ at all redshifts is adopted for Im galaxies, while a constant $S F R$ and $E(B-V)=0.5$ has been assumed for the dusty star-forming galaxies (cf. Paper I). We have then assigned a spectral model to each galaxy according to their $R-K_{\mathrm{s}}$ color and spectral type.

We tested that different assumptions on the $k$-corrections, i.e. by varying model parameters or using the fitting of multiband photometry when available, did not affect significantly our results. For example, the $k$-correction estimates which make use of multi-band fitting of the SED are in excellent agreement with our estimates from $R-K_{\mathrm{s}}$ colors alone, with no systematic shifts in magnitude and a dispersion of about 0.08 in the $J$-band and 0.13 in the $K_{\mathrm{s}}$-band up to $z \sim 1.5$. Varying parameters in the synthetic spectral models, i.e. the IMF (Salpeter 1955; Scalo 1986 and Kennicutt 1983) and the formation redshift $\left(z_{\mathrm{f}}=2,3,6\right)$, the systematic shifts and dispersions at $z<2$ are always less than 0.05 mag. We note that magnitudes derived using the $k$-corrections estimated from observed spectra of local galaxies (Mannucci et al. 2001; Cowie et al. 1994) would be brighter by about $0.1 \mathrm{mag}$ at $z>0.7$, and could be considered upper limits due to the possible evolutionary effect on the galaxy spectrum. We are therefore confident that the derived luminosities are robust with respect to varying this set of assumptions. The resulting effects on the Luminosity Functions induced by changes in our assumptions are all comparable with or smaller than the statistical errors (see Sect. 5).

Figure 4 shows the absolute magnitudes of all galaxies in the spectroscopic sample to $K_{\mathrm{s}}<20$ as derived according to our adopted recipes. For the unidentified or unobserved galaxies we have adopted the photometric redshift $z_{\text {phot }}$. The photometric threshold of the survey, $K_{\mathrm{s}}<20$, is clearly visible in the data and translates into different luminosity limits as a function of redshift, varying from less than $\sim 0.1 L^{*}$ at $z<0.5$ to about $\sim 0.4 L^{*}$ at $z \simeq 1$, assuming $M_{J}^{*}=-23.13+5 \log h_{70}$ and $M_{K_{\mathrm{s}}}^{*}=-24.21+5 \log h_{70}$ (horizontal lines in Fig. 4) for the local LF (Cole et al. 2001). Adopting the mean stellar massto-light ratio and representative stellar mass $\left(\mathcal{M}_{\text {stars }} / L_{K}=1.32\right.$ in solar unit and $\mathcal{M}_{\text {stars }}^{*}=1.44 \times 10^{11} h_{70}^{-2} M_{\odot}$ for the Salpeter IMF) in the local universe (Cole et al. 2001), the limits in luminosities correspond to $\sim 0.1-0.5 \mathcal{M}_{\text {stars }}^{*}$ or even smaller stellar masses since the $\mathcal{M}_{\text {stars }} / L_{K}$ increases as the stellar population ages (Madau et al. 1998).

\section{The estimate of the near-IR Luminosity Function}

After the estimate of the rest-frame near-IR luminosities, we compute the $J$ - and $K_{\mathrm{s}}$-band Luminosity Functions in the following redshift bins: (a) $0.2<z<0.65\left(z_{\text {mean }} \simeq 0.5\right.$ ), (b) $0.75<z<1.3\left(z_{\text {mean }} \simeq 1.0\right)$ and (c) $1.3<z<1.9\left(z_{\text {mean }} \simeq\right.$ 1.5). The redshift bin $0.65<z<0.75$ has been excluded from this analysis because dominated by two groups/clusters (see Fig. 1 and Paper III for details). The first two redshift bins include respectively 132 and 170 galaxies, of which only few objects ( 2 and 21 respectively) do not have a spectroscopic redshift. The highest redshift bin includes a smaller number of galaxies (42) with a high fraction of photometric redshifts $(60 \%)$.

The Luminosity Functions in each redshift bin are estimated using both the $1 / V_{\max }$ formalism (Schmidt 1968; Felten 1976) and the maximum likelihood method STY (Sandage et al. 1979), in order to represent our data with a Schechter (1976) parameterization. In the $1 / V_{\max }$ analysis, for any given redshift bin $\left(z_{1}, z_{2}\right)$ a maximum volume is assigned to each object. This volume is calculated between $z_{1}$ and $z_{\text {up }}$, the latter being the minimum between $z_{2}$ and $z_{\max }$, i.e. the maximum redshift at which this galaxy would have satisfied the magnitude limit $\left(K_{\mathrm{s}}<20\right)$ of the survey. We use the model tracks in order to compute $z_{\max }$. Since the STY method determines the shape of the LF but not its overall normalization, we have normalized the STY LF by matching the galaxy number counts observed in each redshift bin. We have checked and tested the reliability of our results with the independent softwares developed by Zucca et al. (1997) and by Poli et al. (2001), always finding a good agreement between the different methods.

Because of the relatively small number of objects in each redshift bin and only a part of the luminosity function can be recovered, the Schechter parameters $\left(\alpha, M^{*}\right.$ and $\left.\phi^{*}\right)$ derived from our STY analysis are not very well constrained by our data. In particular, the uncertainty on the faint end slope $\alpha$ increases with redshift because of the increase with redshift of the minimum observable luminosity. A sample reaching $K_{\mathrm{s}} \sim 21.0$ 21.5 would be needed to better constrain this parameter at $z>1$. The results will be discussed in Sect. 5 .

\subsection{The treatment of the unidentified objects}

The treatment of the unobserved or spectroscopically unidentified sources is not a major problem because of the high spectroscopic redshift completeness in our survey. Moreover, as discussed in Sect. 2, tested and reliable photometric redshifts were derived for most of the faint unidentified or unobserved objects, leading to an almost negligible number of objects ( 9 in total) without any redshift information (see Paper III).

The small uncertainty introduced by the unobserved or unidentified objects has been addressed in two different ways.

We first use only the spectroscopic sample and take into account the remaining redshift incompleteness (72 galaxies) by applying weights to each galaxy with spectroscopic redshift both in the $V_{\max }$ analysis (Avni \& Bahcall 1980) and in the standard STY formulation (Heyl et al. 1997, cf. also Zucca et al. 1994). The weighting corrections are computed on the 
Table 1. Schechter Luminosity Function parameters $\left(\Phi(M)=0.4 \ln (10) \Phi^{*} 10^{-0.4\left(M-M^{*}\right)(\alpha+1)} \exp \left[-10^{-0.4\left(M-M^{*}\right)}\right]\right)$.

\begin{tabular}{cccccc}
\hline \hline Band & $z$ range & $M-5 \log h_{70}$ range & $\alpha$ & $M^{*}-5 \log h_{70}$ & $\Phi^{*} h_{70}^{-3}\left(10^{-3} \mathrm{Mpc}^{-3}\right)$ \\
\hline$J$ & $0.20-0.65$ & {$[-18.7,-25.2]$} & $-1.22_{-0.20}^{+0.22}$ & $-23.89_{-0.69}^{+0.51}$ & $1.99_{-1.1}^{+1.4}$ \\
$J$ & $0.75-1.30$ & {$[-21.8,-26.3]$} & $-0.86_{-0.44}^{+0.46}$ & $-23.75_{-0.51}^{+0.40}$ & $3.44_{-1.5}^{+1.1}$ \\
$J$ & $1.30-1.90$ & {$[-23.3,-26.3]$} & - & - & - \\
$K_{\mathrm{S}}$ & $0.20-0.65$ & {$[-19.5,-26.0]$} & $-1.25_{-0.20}^{+0.25}$ & $-24.87_{-0.73}^{+0.54}$ & $1.78_{-0.9}^{+1.5}$ \\
$K_{\mathrm{s}}$ & $0.75-1.30$ & {$[-22.7,-27.2]$} & $-0.98_{-0.42}^{+0.47}$ & $-24.77_{-0.55}^{+0.42}$ & $2.91_{-1.4}^{+1.3}$ \\
$K_{\mathrm{s}}$ & $1.30-1.90$ & {$[-24.1,-27.1]$} & - & - & - \\
\hline
\end{tabular}

basis of the fraction of galaxies with spectroscopic redshift in different regions of the $\left(R-K_{\mathrm{s}}\right)-K_{\mathrm{s}}$ plane. This weighting scheme assumes that unidentified objects have the same redshift distribution as the spectroscopically identified with similar $K_{\mathrm{s}}$ magnitudes and $R-K_{\mathrm{s}}$ colors. While this scheme can be reasonably applied over most of the $\left(R-K_{\mathrm{s}}\right)-K_{\mathrm{s}}$ plane, the weights are highly uncertain for the optically faintest and reddest galaxies, most of which do not have a spectroscopic redshift. Since the photometric redshifts of these objects are statistically higher than those sampled by the measured spectra, this method would not allow to correctly estimate the luminosity function in the highest redshift bin.

As a second approach, we therefore base the "best" estimate of the luminosity function on the sample of galaxies with spectroscopic plus photometric redshifts. In this case, the $9 \mathrm{ob}-$ jects without any redshift information (because of the absence of the extended multi-band photometry) have an almost negligible effect. To account for them we correct the STY normalization (from $1 \%$ to $8 \%$ in the 3 redshift bins) according to the fraction of such objects in each bin. The results from the two methods (Figs. 5 and 6) are completely consistent with each other in the first two redshift bins, while some discrepancies are present in the highest redshift bin because, as mentioned above, the weighting correction function is unable to properly take into account the optically faintest and reddest galaxies. Furthermore we tested that the uncertainties in the photometric redshifts did not affect significantly our results. We extracted random samples from the original photometric redshift catalog using the measured dispersion. The derived fluctuations in each magnitude bin of the LFs resulted smaller than Poisson errors and in general the simulated LFs are consistent with the "best" estimate also in the higher redshift bin with no significant systematic effects. In the following sections we will discuss the "best" LFs derived using spectroscopic plus photometric redshifts.

\section{The cosmic evolution of the near-IR Luminosity Function}

Thanks to the high statistical significance and completeness of our sample it is possible to investigate the evolution of the nearIR LF over a wide range of cosmic time. Figs. 5 and 6 show the $1 / V_{\max }$ and the maximum likelihood (STY) results for the LFs in the selected redshift bins and in the $J$ - and $K_{\mathrm{S}}$-bands, respectively.

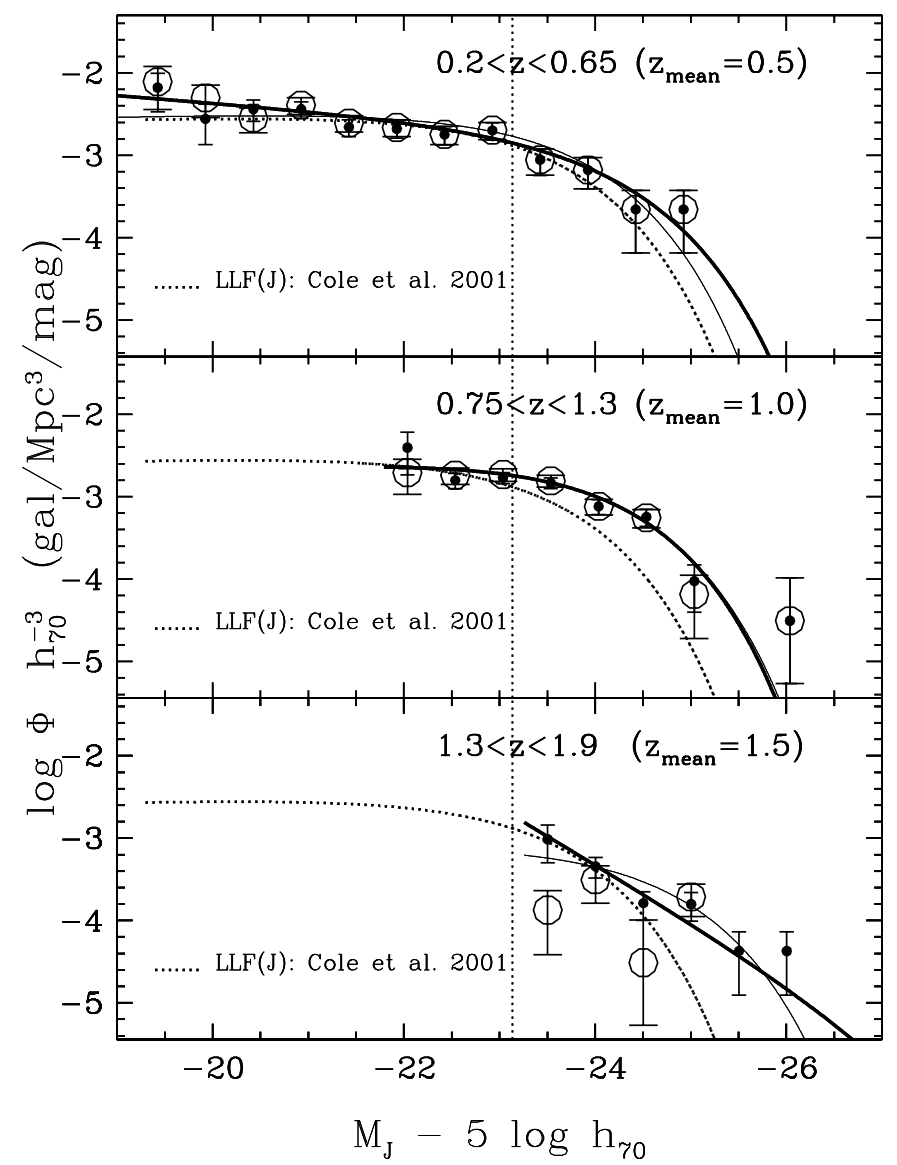

Fig. 5. The rest-frame $J$-band Luminosity Function in the redshift bins: $z_{\text {mean }} \simeq 0.5$ (top panel) $z_{\text {mean }} \simeq 1.0$ (middle panel) and $z_{\text {mean }} \simeq 1.5$ (bottom panel). Points have been derived from $1 / V_{\max }$ analysis (open circles using only spectroscopic $z$ and weighted incompleteness corrections, while filled small dots using both spectroscopic and photometric $z$, see Sect. 4.1), while solid curves are the LF Schechter fits derived from the STY maximum likelihood analysis (thin solid lines are the fits obtained fixing the $\alpha$ parameter at the local value, see text). The dotted curves and vertical dotted lines show the local LF in the $J$-band and $M_{J}^{*}$ at $z=0$ from Cole et al. (2001).

We find that the Luminosity Functions are fairly well fitted by Schechter functions in the first two redshift bins. The magnitude ranges and the best-fit Schechter parameters are summarized in Table 1, with the uncertainties derived from the projection of the $68 \%$ confidence ellipse. In the highest redshift bin, due to the bright and limited range in magnitude, our data 


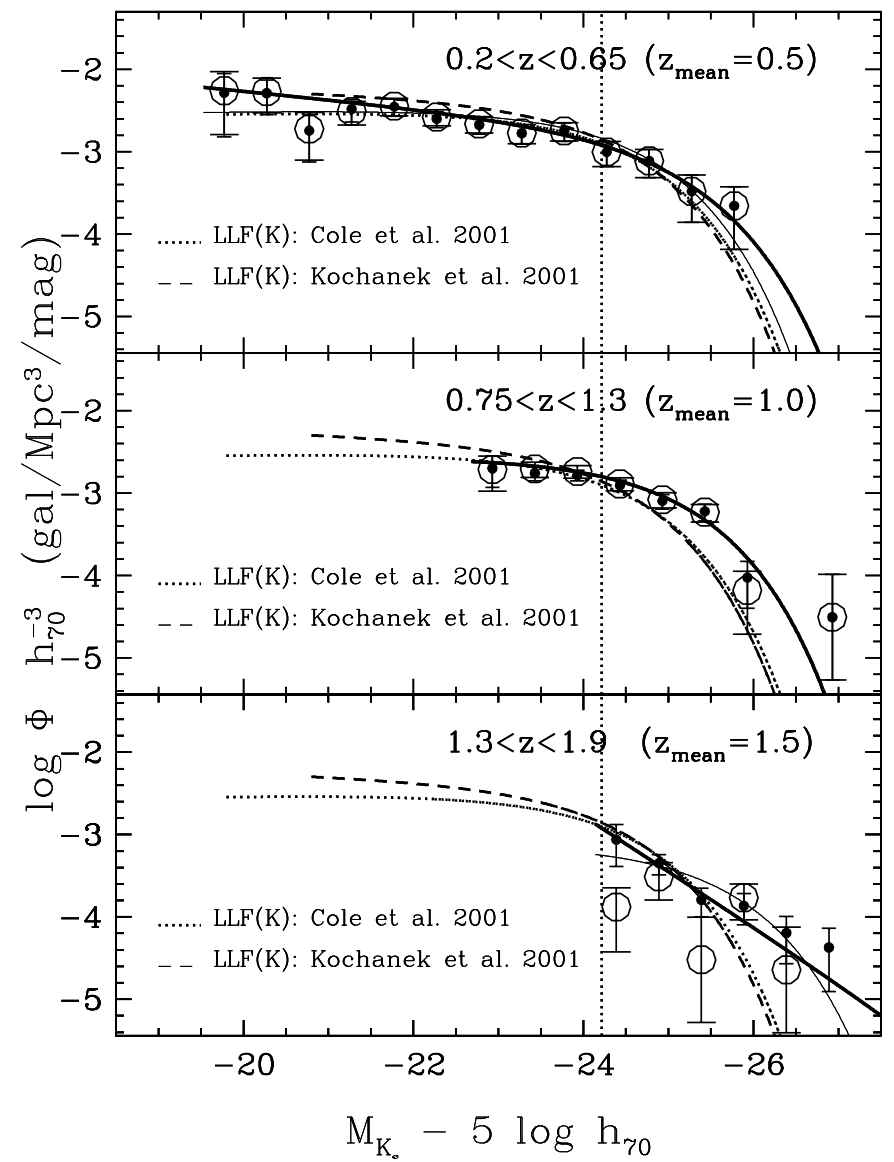

Fig. 6. The same as in Fig. 5, but for the rest-frame $K_{\mathrm{s}}$-band. The dotted curves and vertical dotted lines show the local LF in the $K_{\mathrm{s}}$-band and $M_{K}^{*}$ at $z=0$ from Cole et al. (2001), while dashed curves show the local LF from Kochanek et al. (2001).

Table 2. Galaxy luminosity evolution at different redshift.

\begin{tabular}{cccc}
\hline \hline Band & $\Delta M(z=0.5)$ & $\Delta M(z=1.0)$ & $\Delta M(z=1.5)$ \\
\hline$J$ & $-0.23_{-0.17}^{+0.16}$ & $-0.69_{-0.12}^{+0.12}$ & $-1.17_{-0.24}^{+0.22}$ \\
$K_{\mathrm{s}}$ & $-0.11_{-0.18}^{+0.17}$ & $-0.54_{-0.13}^{+0.12}$ & $-1.07_{-0.27}^{+0.23}$ \\
\hline
\end{tabular}

are well fitted with a single power law $\left(\alpha \simeq-2.7_{-0.7}^{+0.5}\right)$. This does not mean that a Schechter function can not be a good representation of the luminosity function also in this redshift bin. This is shown by the thin solid lines in the lower panels of Figs. 5 and 6 , which represent fits with a Schecther function obtained by fixing the faint end slope $\alpha$ at the local value. However, because of the bright limit in absolute magnitude at these redshifts, which reduces significantly the range of sampled luminosities, the Schechter parameters are very poorly determined by these data. For this reason the Schechter parameters for this redshift bin are not reported in Table 1. We point out that a direct comparison of the Schechter parameters could be quite misleading because the parameters are correlated.

In order to trace the evolution down to $z \sim 0$, we compared the observed LFs as derived from our sample to the Local Luminosity Function (LLF) by Cole et al. (2001) in the $J$ and $K_{\mathrm{s}}$ bands (Figs. 5, 6) and by Kochanek et al. (2001) in the $K_{\mathrm{s}}$-band. We then explored two possible evolutionary scenarios: luminosity or density evolution.

Our analysis (both $V_{\max }$ and STY) shows that the data (Figs. 5 and 6) are consistent with a mild evolution from $z=0$ to $z \simeq 1.5$ both in $J$ - and $K_{\mathrm{s}}$-bands. In particular the faint-end of the LFs $\left(L<L^{*}\right)$ is consistent with the local estimates, with no statistically significant evidence for a change either in the slope or normalization up to $z<1.3$ (consistently with Cohen 2002). At higher redshift this part of the luminosity function is not well sampled by our data, but also in this bin a Schechter function with local faint-end slope is consistent with the data (Figs. 5 and 6). Viceversa, it is interesting to note that the density of luminous galaxies (e.g. $M_{K_{\mathrm{s}}}-5 \log h_{70}<-25.5$ ) is significantly higher than locally.

Because of the statistical correlation between the Schechter parameters $\left(\alpha, M^{*}\right.$ and $\left.\Phi^{*}\right)$, in order to estimate the luminosity and/or density evolution within our sample and in comparison with the local LFs, we have fixed the $\alpha$ parameters of our LFs to the same values observed locally (Cole et al. 2001), $\alpha_{J}=-0.93$ and $\alpha_{K_{\mathrm{s}}}=-0.96$ (thin solid lines in Figs. 5, 6). We are allowed to do this because all our best fit $\alpha$ values are consistent with the local ones (see Table 1). Assuming local $M_{J}^{*}=-23.13+5 \log h_{70}$ and $M_{K_{\mathrm{s}}}^{*}=-24.21+5 \log h_{70}$ from Cole et al. (2001) we find a luminosity evolution at $z \simeq 1$ of the order of $\Delta M_{J} \simeq-0.69 \pm 0.12$ and $\Delta M_{K} \simeq-0.54 \pm 0.12$ with the normalizations $\left(\phi^{*}\right)$ in the first two redshift bins consistent with the local values, within from 3 to $20 \%$. At $z>1.3$ there is an indication of an even higher luminosity evolution $\left(\Delta M_{J} \simeq-1.17\right.$, $\Delta M_{K} \simeq-1.07$ ), while the $\phi^{*}$ values decrease by a factor $4-5$ with respect to the local value. This last result should be taken with caution because of the lower statistics in the highest redshift bin and the smaller range in luminosity covered.

The luminosity evolution estimated from STY analysis are summarized in Table 2 with the formal statistical $1 \sigma$ confidence limits. The uncertainties introduced by the $k$-correction, discussed in Sect. 3, are always less than the statistical errors, and less than 0.03 and 0.10 mag at $z<1.3$ and $z \simeq 1.5$, respectively.

We recall here that Cimatti et al. (2002b) discussed the photometric selection effects present in the K20 sample and showed that, on average, the total flux of spirals and ellipticals with $L \leq L^{*}$ are underestimated by 0.1 and 0.25 mag respectively, while at higher $L$ the flux lost for elliptical galaxies could be even higher. For this reason we are confident that our estimate of a positive luminosity evolution is quite conservative. The above estimates of the luminosity evolution could increase on average by about -0.2 mag if we take into account this underestimate of the total flux.

\section{The Luminosity Function by spectral or color type}

In order to investigate the role of different galaxy populations, we divide the K20 sample in two subsamples on the basis of the spectroscopic classification, i.e. early type galaxies without strong emission lines and late type galaxies with emission lines, and study the near-IR LF for the different populations in the two redshift bins at $z_{\text {mean }} \simeq 0.5$ and 1 . The high fraction of photometric redshifts without any spectroscopic information 


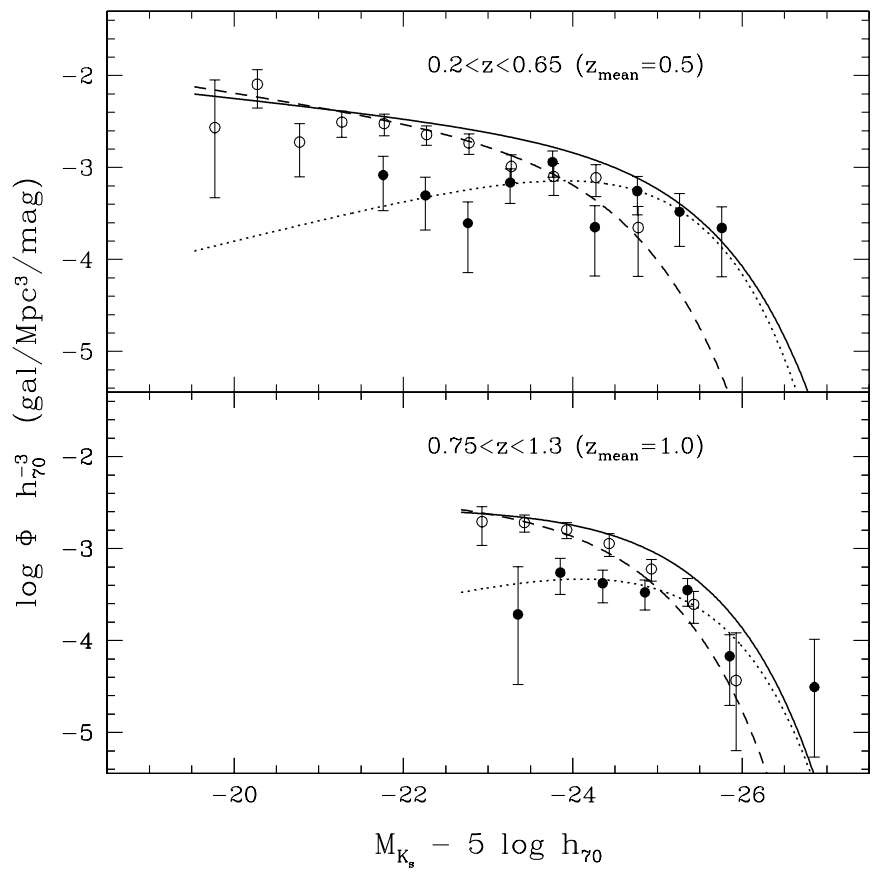

Fig. 7. Rest-frame $K_{\mathrm{s}}$-band Luminosity Function in two redshift bins: $z_{\text {mean }} \simeq 0.5$ (top panel) $z_{\text {mean }} \simeq 1.0$ (bottom panel). Points and lines derive from $1 / V_{\max }$ and STY analysis for early (filled symbols and dotted lines) and late (open symbols and dashed lines) galaxies respectively. The solid curves are the STY LF for the total sample in the $K_{\mathrm{s}}$-band as in Fig. 6.

does not allow us to extend such studies to the highest redshift bin. We take into account the spectral incompleteness using two weighting functions (see Sect. 4.1) derived for each spectral type and correcting the overall normalization in the STY method using the color information of unclassified objects.

The results are shown in Fig. 7. Our analysis indicates that the faint-end slope of the LFs at these redshifts shows the same general dependence on galaxy spectral type as that found in numerous studies of the local LF. Specifically, the faint-end slope is much steeper for late/emission line galaxies than for early type galaxies. A similar result has been found by Cohen (2002) in the HDF-north. More important, we find, for the first time, that at $z_{\text {mean }} \simeq 0.5$, early type galaxies clearly dominate the bright-end of the Luminosity Function, in agreement with local observations (Kochanek et al. 2001). A similar result is also visible at $z_{\text {mean }} \simeq 1.0$. Some of the previous surveys, selected in the optical bands with follow-up $K$-band observations (e.g. Cohen 2002), present a bias against identifying red early type galaxies at high redshift because of substantial incompleteness of EROs in the samples.

The overall density of early type galaxies at $z \simeq 0.5$ is consistent with the local estimate (Kochanek et al. 2001), while at $z \simeq 1$, their number density shows at most a small decrease $(<30 \%)$, consistent with recent results from Im et al. (2002). This decrement could be due both to the existence of a population of galaxies morphologically classified as early-type but with blue colors due to some recent episodes of star formation

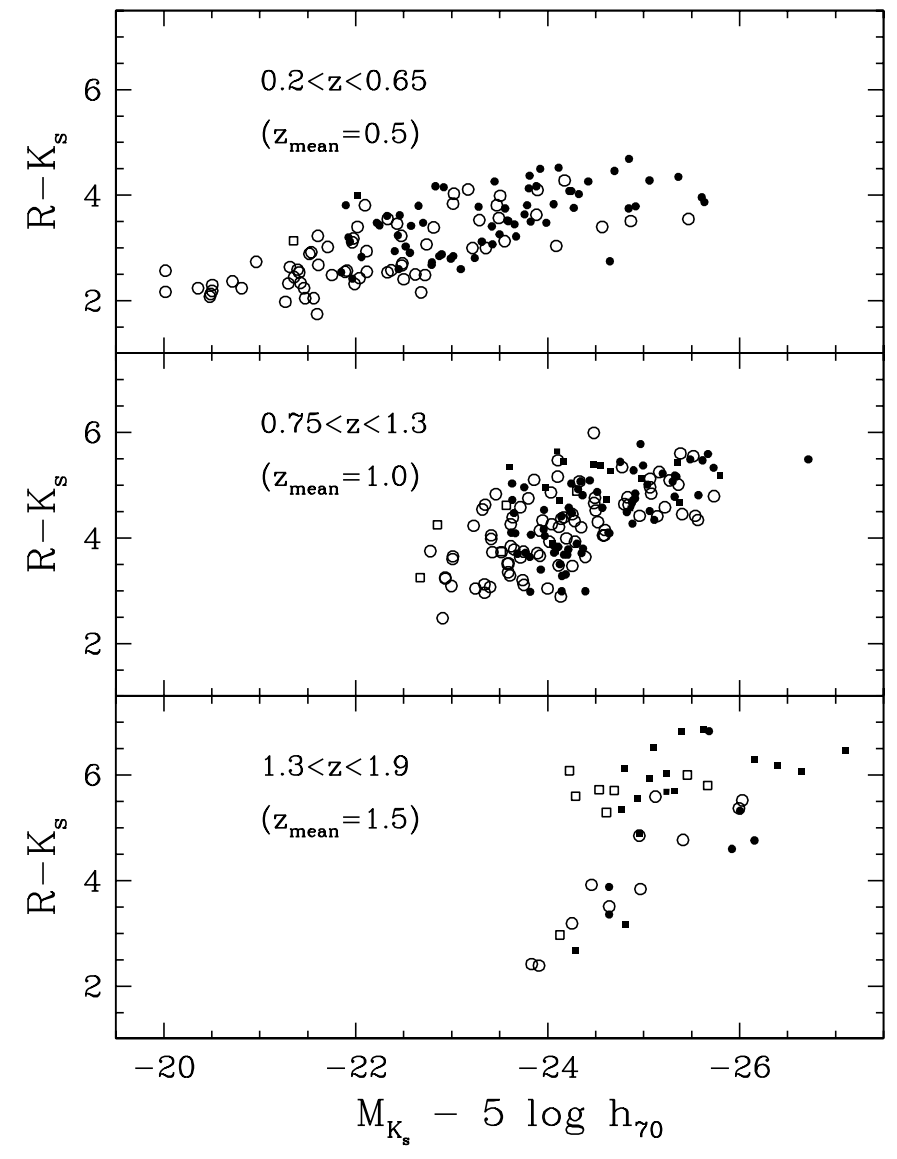

Fig. 8. $R-K_{\mathrm{s}}$ colors vs. rest-frame absolute $K_{\mathrm{s}}$ magnitudes for K20 galaxy sample in 3 different redshift bins. Circles represent galaxies identified spectroscopically, while squares are unidentified or unobserved galaxies plotted at $z=z_{\text {phot }}$. In each redshift bin empty and filled symbols refer to galaxies with $z<z_{\text {mean }}$ and $z \geq z_{\text {mean }}$, respectively.

(Menanteau et al. 2001; Im et al. 2001) and to our spectral incompleteness, more severe for red/early type galaxies.

Similar results are obtained if we divide the galaxies in two samples according to their $R-K_{\mathrm{S}}$ colors (we adopt the color of Sa galaxies to divide the sample in red and blue).

We conclude that red and early type galaxies dominate the bright-end of the Luminosity Function already at early epoch and that their number density shows at most a small decrease $(<30 \%)$ up to $z \simeq 1$.

Figure 8 shows the optical/near-IR colors versus near-IR luminosities in the three redshift bins. While the magnitude limit of the survey, $K_{\mathrm{s}}<20$, corresponds to different low luminosity limits in the different redshift bins, there appears to be a correlation of the optical/near-IR colors with near-IR luminosities. In first approximation this correlation can be interpreted as a correlation of age and/or specific star formation rate with stellar mass, with the most "massive" galaxies being "old" and the "low-mass" galaxies being instead dominated by young stellar populations. This is now well established for local galaxies (Gavazzi et al. 1996; Boselli et al. 2001; Kauffmann et al. 2002) and we extend it to $z \sim 1.5$. Figure 8 (see empty and filled circles representing galaxies with $z<z_{\text {mean }}$ and $z \geq z_{\text {mean }}$ ) shows 
Table 3. luminosity density ${ }^{a}$ at different redshift.

\begin{tabular}{|c|c|c|c|c|}
\hline redshift & $\log \rho_{J}^{b}$ & $\log \rho_{J}^{c}$ & $\log \rho_{K_{\mathrm{s}}}{ }^{b}$ & $\log \rho_{K_{\mathrm{s}}}{ }^{c}$ \\
\hline 0.5 & 20.20 & $20.20_{-0.02}^{+0.01}$ & 20.19 & $20.17_{-0.02}^{+0.01}$ \\
\hline 1.0 & 20.25 & $20.28_{-0.03}^{+0.05}$ & 20.21 & $20.26_{-0.03}^{+0.05}$ \\
\hline 1.5 & $>19.90$ & - & $>19.88$ & - \\
\hline
\end{tabular}

${ }^{a}$ In $h_{70} \mathrm{~W} / \mathrm{Hz} / \mathrm{Mpc}^{3}$ units.

${ }^{b}$ Observed.

${ }^{c}$ LF-corrected (see text).

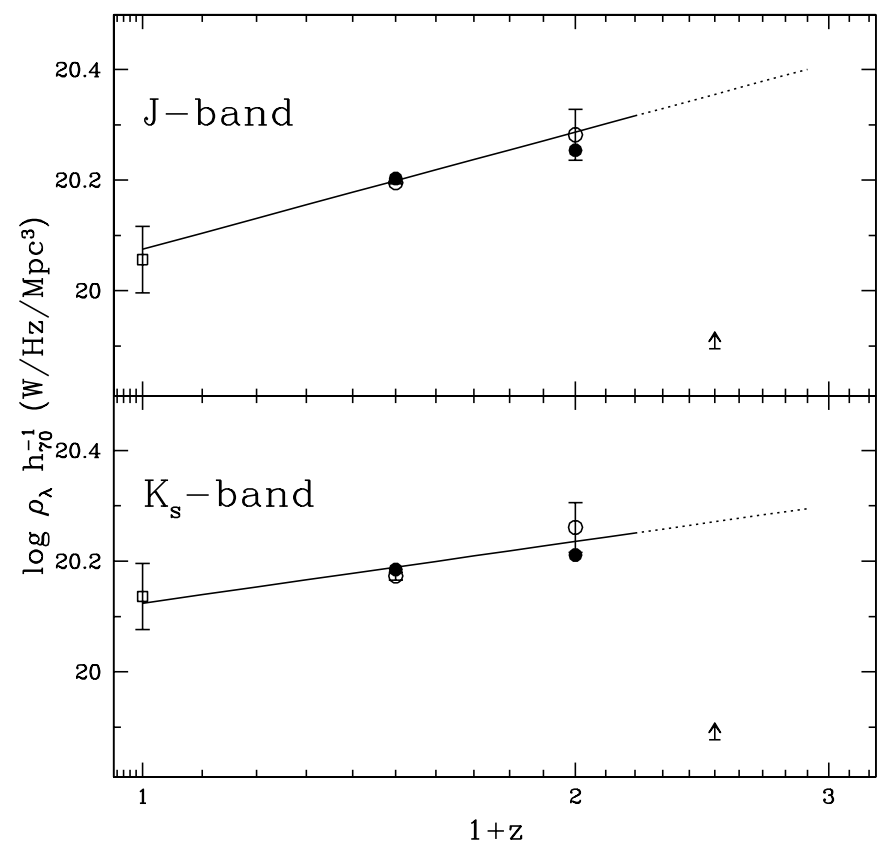

Fig. 9. Comoving luminosity density in $J-$ (top panel) and $K_{\mathrm{s}}$-bands (bottom panel). The filled circles are the value derived directly from observations (see text), while open circles are the "LF-corrected" estimates. The solid lines show the best-fit power laws. The open squares are from Cole et al. (2001).

that the observed correlation is not induced by a redshift effect within the bins. In Sect. 8 we compare the observed correlation with HMM predictions.

\section{The near-IR luminosity density evolution}

Tracing the cosmic emission history of the galaxies at different wavelengths offers the prospect of an empirical determination of the global evolution of the galaxy population. Indeed, it is independent of the details of galaxy evolution and depends mainly on the star formation history of the universe (Lilly et al. 1996; Madau et al. 1998). Various attempts to reconstruct the cosmic evolution of the comoving luminosity density have been made previously mainly in the UV and optical bands (Lilly et al. 1996; Cowie et al. 1999). Our survey offers the possibility to investigate it in the near-IR using a LF extended over a wide range in luminosity.

We estimate the comoving luminosity density directly from observed sources using the $V_{\max }$ formalism (see for details Lilly et al. 1996). The major source of uncertainty is the contribution from galaxies fainter than the magnitude limit $K_{\mathrm{s}}<20$. We have therefore estimated also the "LFcorrected" luminosity density using the best-fit LF from STY method and extending it to fainter luminosity (we adopt $0.02 L^{*}(z)$ as our lower limit in luminosity for this extrapolation). A formal uncertainty in this procedure was estimated by considering the range of acceptable Schechter parameters values (see Table 1).

Figure 9 shows luminosity densities derived in $J$ - and $K_{\mathrm{s}}$ band as a function of redshift. Up to $z<1.3$ the uncertainties due to faint galaxies and LF parameters are small. At higher redshift the uncertainties in the LF parameters do not allow to constrain the luminosity density values. We could therefore only estimate a lower limit to the luminosity density using the observed value.

We find a slow evolution with redshift of the observed nearIR luminosity density, consistent with the results by Cohen (2002) in the HDF-north, even if she had to apply a large correction to her data because of the disappearance of absorption galaxies at high redshift in her sample as they became EROs. Using the observed local luminosity densities derived from Cole et al. (2001), the luminosity density evolution up to $z \leq 1.3$ is well represented by a power law, $\rho_{\lambda}(z)=\rho_{\lambda}(z=$ $0)(1+z)^{\beta(\lambda)}$. We find $\beta(J) \simeq 0.70$ and $\beta\left(K_{\mathrm{s}}\right) \simeq 0.37$ (Fig. 9).

The near-IR luminosity density evolution is much slower than that found in the UV and optical bands $(\beta=3.9-2.7$ from 0.28 to $0.44 \mu \mathrm{m}$ by Lilly et al. 1996 and $\beta=1.5$ at 0.15 , $0.28 \mu \mathrm{m}$ by Cowie et al. 1999 , for $\Omega_{m}=1$ ). Indeed, while the optical luminosity density evolution is mainly related to the star formation history, the evolution of the near-IR luminosity density is more closely related to the stellar-mass density. The local stellar mass density, derived by Cole et al. (2001) is $\Omega_{\text {stars }}=(3.7 \pm 0.6) \times 10^{-3} h_{70}^{-1}$. If we adopt the stellar massto-light ratio for the galaxy spectral models which best match colors and spectral types for each galaxy, the mean $\mathcal{M}_{\text {stars }} / L_{K}$ in our sample becomes 0.63 and 0.54 at $z=0.5$ and 1 , with small variations due to the model parameters adopted (see Sect. 3). With these values of $\mathcal{M}_{\text {stars }} / L_{K}$ we derive $\Omega_{\text {stars }}=$ $(2.0 \pm 0.1) \times 10^{-3} h_{70}^{-1}$ and $\Omega_{\text {stars }}=(2.1 \pm 0.3) \times 10^{-3} h_{70}^{-1}$ at $z=0.5$ and 1 respectively. This analysis suggests that the evolution of the stellar mass density is relatively slow with redshift, with a decrease of about a factor $1.8 \pm 0.4$ from $z=0$ to $z \simeq 1$, consistently with recent results from Dickinson et al. (2002a).

At $z>1$ we can only give a lower limit to the near-IR luminosity density. As discussed by Madau et al. (1998) (cf. also Pozzetti \& Madau 2001), a robust determination of the near-IR luminosity density at $z>1$ could be fundamental to disentangle between different cosmic histories of star formation (SFH). In fact, SFHs peaked at intermediate redshift $(1<z<2)$ predict a decrease in the near-IR luminosity density at $z>1-$ 2 , while if $50 \%$ of the stars formed at $z>2$ (similar to the PLE adopted here), the corresponding near-IR luminosity density flattens at $z>1$ without a strong decrease (cf. Fig. 6 in Pozzetti \& Madau 2001). A detailed comparison of luminosity and stellar-mass density with model predictions and empirical star formation histories will be further investigated in a forthcoming paper. 

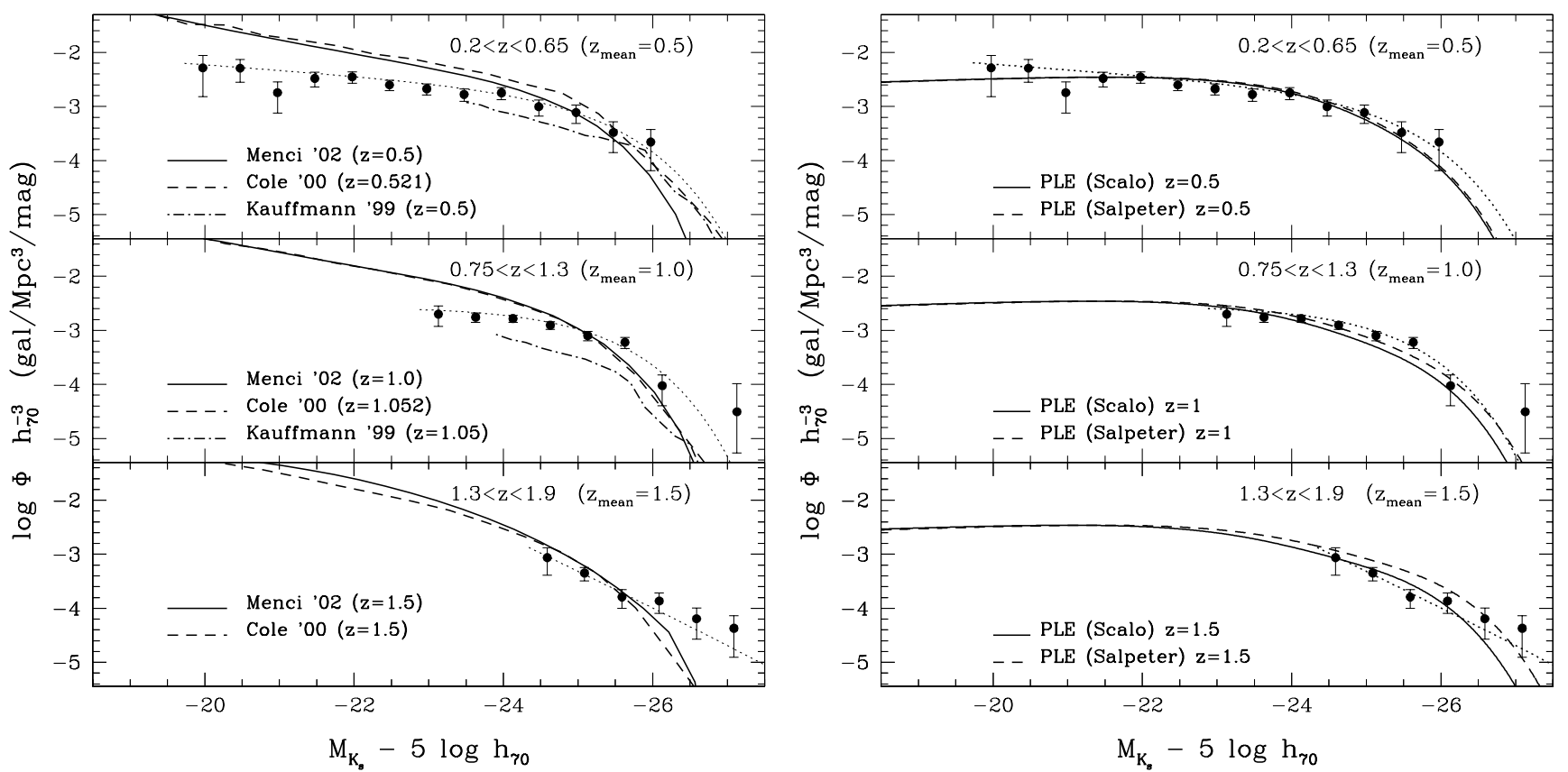

Fig. 10. The rest-frame $K_{\mathrm{s}}$-band Luminosity Function in the redshift bins: $z_{\text {mean }} \simeq 0.5$ (top panels) $z_{\text {mean }} \simeq 1.0$ (middle panels) and $z_{\text {mean }} \simeq 1.5$ (bottom panels) compared to PLE (right panels) and HMM (left panels) models. We have used the models closer to $z_{\text {mean }}$ or interpolated between different redshifts. Data points derive from $1 / V_{\max }$ analysis, while dotted curves are the LF Schechter fit derived from STY analysis. We have corrected the data on average by -0.2 magnitude because photometric selection effects (see text).

\section{The comparison with model predictions}

We have compared our LF with the predictions from two competitive scenarios of galaxy evolution: the Hierarchical Merging Models (HMMs) and the Pure Luminosity Evolution (PLE) (Fig. 10). For the HMMs, we used the predictions by Cole et al. (2000, C00, kindly provided us by C. Baugh), Menci et al. (2002, M02), and GIF (Kauffmann et al. 1999). For the GIF simulations, which combine the large high-resolution $\mathrm{N}$-body simulations with semi-analytical models, we derive the $\mathrm{LF}$ at a magnitude above which it can be considered complete in luminosity given the mass limit of the simulated galaxy sample $\left(2 \times 10^{10} h_{70}^{-1} M_{\odot}\right)$. For the PLE parameterization, we adopted the predictions of Pozzetti et al. (1996, 1998, PPLE) (for details see also Paper IV).

For a more reliable comparison with models, we corrected the observed magnitudes by $-0.2 \mathrm{mag}$, in order to take into account the average loss of total flux in the K20 sample (see Papers III-IV).

As discussed in Paper IV at low- $z(z=0.5)$ the HMMs by Cole et al (2000) and by Menci et al. (2002) predict many more galaxies than observed (cf. Fig. 3 in Paper IV). From the present study (upper panel of Fig. 10) we can therefore conclude that such excess is due to low luminosity, "low-mass" objects, which dominate the steep faint-end in the HMM LF. At $z=1$, the HMMs predict even steeper LFs because low-mass galaxies become more numerous in the hierarchical scenario at higher redshift. This is in contrast with the LF derived from our data at $z=1$, which does not show a similar behaviour. These discrepancies are consistent with previous results both in the local universe (cf. Fig. 1 in Baugh et al. 2002) and at high redshift $(z<1$ and $z \sim 3$ ) for the $B$ and UV rest-frame LF, respectively (Poli et al. 2001; Somerville et al. 2001).
Also at the bright end of the LF the HMMs appear to be in disagreement with our data at $z \simeq 1$, where they underpredict the density of bright $\left(M_{K_{\mathrm{s}}}<-25.5+5 \log h_{70}\right)$ galaxies with respect to our data. For example, our data at $M_{K_{\mathrm{s}}}=$ $-25.6+5 \log h_{70}$ and $z \simeq 1$ are about a factor 2.6 higher than the HMM predictions and even higher at brighter magnitudes if we consider the STY maximum likelihood fit. In the highest redshift bin the excess over the predictions becomes significant at $M_{K_{\mathrm{s}}}<-26+5 \log h_{70}$. In comparison, the GIF simulated catalogue at $z=1$ underpredicts the K20 LFs at all magnitudes. As discussed by Kauffmann et al. (1999), the GIF model produces a factor 2-3 too few galaxies at magnitudes around $L^{*}$ already at $z=0$, while it produces an excess of bright local galaxies. This problem with respect to the local LF affects also the Cole et al. (2000) model (see Fig. 4 in Baugh et al. 2002). Therefore, as discussed in Sect. 5, while the density of luminous objects $\left(M_{K_{\mathrm{s}}}-5 \log h_{70}<-25.5\right)$ is quite constant or mildly increasing with $z$ within our sample, and higher than the local density, it is rapidly decreasing in the Cole et al. (2000) and GIF models at $z>0.5$ (Fig. 11) in clear conflict with our data. The problem of a negative evolution with redshift of luminous objects does not affect instead the Menci HMM model, even if also this model significantly and systematically underpredicts their number density at all redshifts.

In addition to the comparison of the data and predictions for the LF, it is also important to verify whether the bright $L>L^{*}$ galaxies in the K20 survey have colors consistent with the HMM predictions. Figure 12 shows the comparison between the $z=1.05 \mathrm{GIF}$ simulated catalogue and data at $0.75<z<1.3\left(z_{\text {mean }} \simeq 1\right)$. While the deficiency of simulated blue low-luminosity $\left(R-K_{\mathrm{s}}<4.2, M_{K_{\mathrm{s}}}-5 \log h_{70}>-23.5\right)$ galaxies is due mainly to the mass limit of the GIF catalogue, a 


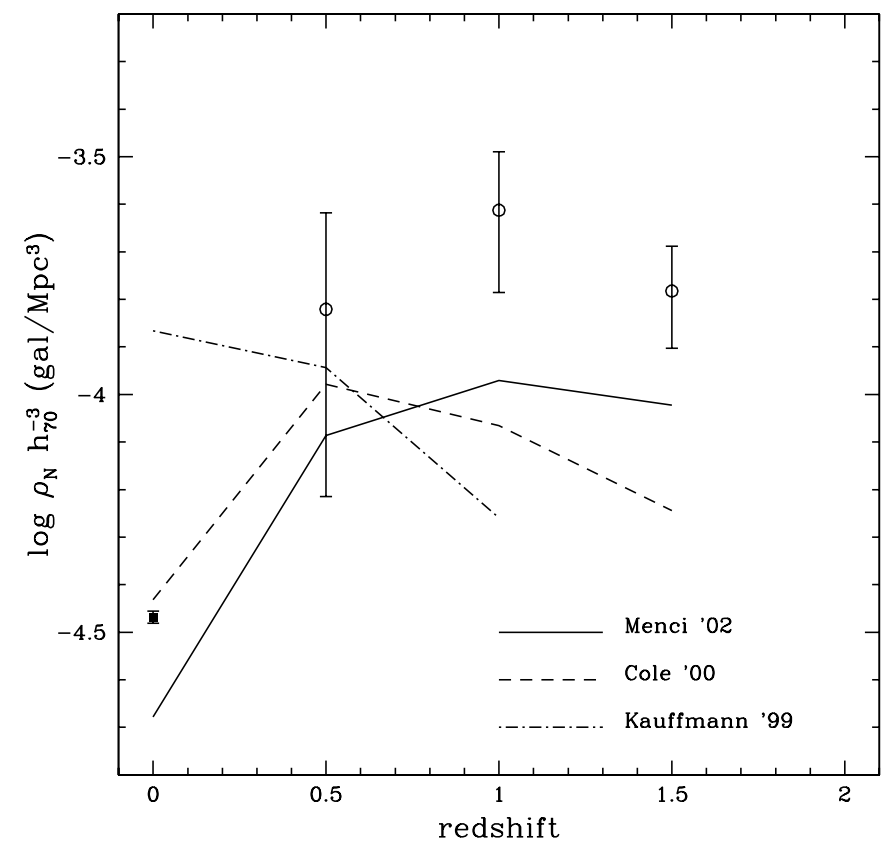

Fig. 11. Comoving number density of luminous galaxies $\left(M_{K_{\mathrm{s}}}<\right.$ $\left.-25.5+5 \log h_{70}\right)$ in the $K_{\mathrm{s}}$-bands. The open circles are the values derived directly from observations, while the filled square is from Cole et al. (2001). The different lines show models as in Fig. 10.

serious discrepancy emerges in the two distributions in the magnitude range where the GIF catalogue is expected to be complete. The GIF simulated catalogue shows an excess of blue high-luminosity galaxies $\left(R-K_{\mathrm{s}}<4.4, M_{K_{\mathrm{s}}}-5 \log h_{70}<\right.$ -24.5) and a deficiency of red luminous galaxies (see also the color distributions of high-luminosity galaxies, $M_{K_{\mathrm{s}}}-$ $5 \log h_{70}<-24.5$, in the right panel of Fig. 12, normalized to the same comoving volume). This result is in agreement with the finding of a deficiency of EROs in the HMMs (Daddi et al. 2002; Firth et al. 2002; Smith et al. 2002), but, since it is based not only on colors but also on the luminosity of the galaxies, it points out in particular a clear deficiency of red luminous galaxies in the HMM predictions. This plot clearly shows that a comparison of LF and colors is much more powerful than that of the colors or LF alone and that the GIF $K_{\mathrm{s}}$-band LF at $z=1$ is obtained with a radically different mix of galaxies compared to the observed one. While the bright end of the HMM LF at $z=1$ is dominated by actively starforming galaxies, in the K20 sample it is dominated by the passively evolving galaxies. Given that blue galaxies suggest younger stellar population and lower $\mathcal{M}_{\text {stars }} / L_{K}$ ratios, we expect that the failure of this model in reproducing our LF (see Fig. 10) should be even stronger in term of density of "massive" galaxies at $z \sim 1$ (cf. also the luminosity and stellar-mass functions in Baugh et al. 2002).

The right panels of Fig. 10 show the comparison of our data with the PLE predictions, computed with two different IMF (Scalo and Salpeter). The overall agreement is much better than with the HMM predictions even if some discrepancies, but at much lower level of significance, are present. In particular, in the highest redshift bin the parameterization with the Salpeter IMF overpredicts the total number of galaxies (see also Paper IV), while the Scalo IMF

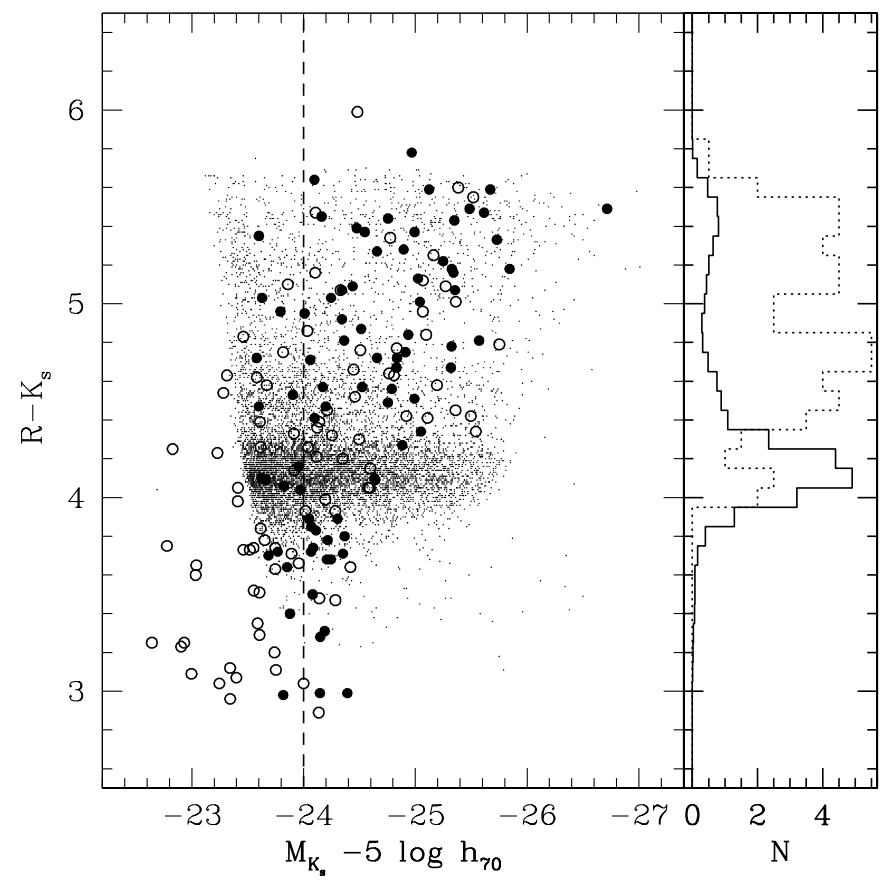

Fig. 12. Left panel: $R-K_{\mathrm{s}}$ colors vs. rest-frame absolute $K_{\mathrm{s}}$ magnitudes for $z=1.05$ GIF simulated catalogue (small dots) and data (circles) at $0.75<z<1.3\left(z_{\text {mean }} \simeq 1\right.$ ) (empty and filled circles refer to $z<1$ and $z>1$ respectively). Vertical dashed line represents approximately the completeness magnitude limit of GIF catalogue corresponding to its mass limit (see text). Right panel: color distribution of high-luminosity galaxies $\left(M_{K_{\mathrm{s}}}-5 \log h_{70}<-24.5\right)$ observed (dotted line) and simulated (continue line), normalized to the same comoving volume.

(dominated by low/intermediate mass stars, which induce a lower luminosity evolution compared to Salpeter at $z>1$ ) fits well the density of galaxies at $M_{K_{\mathrm{s}}}>-26.0+5 \log h_{70}$, but falls below the data for the brighter galaxies. In Paper IV we found that in the context of PLE models also the observed redshift distribution of K20 galaxies is better fitted assuming an IMF dominated by low/intermediate mass stars (e.g. Scalo IMF) than a Salpeter IMF (see also Pozzetti et al. 1996; Broadhurst \& Bouwens 2000). PLE models with a flatter IMF (i.e. Salpeterlike) can be made consistent with the data (redshift distribution and LF) only if the rapid evolution induced by massive stars is sufficiently suppressed by dust attenuation (Totani et al. 2001, see Paper IV). Indeed, such a dusty phase is predicted by many models for the formation of high redshift spheroids and could be associated to high redshift SCUBA sources.

\section{Summary and discussion}

The cosmic evolution of the $K_{\mathrm{s}}$-band selected field galaxy population has been studied over the redshift interval $0.2<z<1.9$ using about 500 galaxies from the K20 spectroscopic survey. The sample spans a wide range in redshift and look-back time and allows to study the evolution of the near-IR Luminosity Function both within the sample and in comparison to the local population.

We take advantage of the near-IR selection (in particular in the $K$-band), in which the $k$-corrections are relatively invariant 
to galaxy type and relatively small also at high redshift (Cowie et al. 1994), and the dust extinction effects are less severe than in optical samples.

We derived the near-IR luminosity function in the restframe $J$ and $K_{\mathrm{s}}$-band in three redshift bins $\left(z_{\text {mean }} \simeq\right.$ $0.5,1.0,1.5)$. The detailed analysis of the observed LF at different redshifts and the comparison with the local LF and with the predictions of galaxy formation models provided the following results:

1- A mild evolution is observed both in the $J$ and $K_{\mathrm{s}}$ Luminosity Functions to $z \simeq 1.5$, in agreement with previous indications by Cowie et al. (1996), Cohen (2002), and Feulner et al. (2003). There is no evidence of a steepening of the faint-end LFs up to $z \sim 1.3$. In particular, the faint-end $\left(L<L^{*}\right)$ is consistent with the local estimates up to $z<1.3$, while at the bright end the data show a luminosity evolution of about $\Delta M_{J} \simeq-0.69 \pm 0.12$ and $\Delta M_{K} \simeq-0.54 \pm 0.12$ at $z \simeq 1$. The density of luminous galaxies $\left(M_{K_{\mathrm{s}}}-5 \log h_{70}<-25.5\right)$ is relatively constant or mildly increasing with redshift within our sample and higher than locally at all redshifts.

2- Pure density evolution cannot reproduce the observed LF at $z \leq 1.3$.

3 - Red and early-type galaxies clearly dominate the brightend of the Luminosity Function at $z \simeq 0.5$ and a similar trend is also visible at $z \simeq 1$, showing that such systems were already in place and fully assembled at that cosmic epoch. Their number density shows at most a small $(<30 \%)$ decrease up to $z \simeq 1$.

4- The evolution of the rest-frame near-IR comoving luminosity densities up to $z \simeq 1$ can be described by power laws, $\rho_{\lambda}(z)=\rho_{\lambda}(z=0)(1+z)^{\beta(\lambda)}$, with $\beta(J) \simeq 0.70$ and $\beta\left(K_{\mathrm{s}}\right) \simeq 0.37$. Such an evolution is much slower than those observed in the UV and optical bands.

5- The hierarchical merging models overpredict the LF at low luminosity at all redshifts, whereas they underpredict the density of high-luminosity galaxies at $z>1$. HMMs by Kauffman et al. (1999) and Cole et al. (2000) overpredict high luminosity galaxies at $z=0$, and predict a negative density evolution of the bright end of the LF at $z>0.5$ which is not observed.

6- The PLE predictions are in rather good agreement with the mild luminosity evolution observed up to $z \simeq 1.5$.

7- There appears to be a clear correlation of the optical/near-IR colors, i.e. in first approximation the specific star formation rate and ages, with near-IR luminosities, i.e. the stellar mass. This correlation suggests that the most "massive" galaxies are "old", while the "low-mass" galaxies are instead dominated by young stellar populations. The GIF model shows instead a clear deficiency of red luminous galaxies at $z \sim 1$ compared to the observations.

From the analysis of the LF and of the comoving near-IR luminosity density, we can derive some indications on the evolution of the Galaxy Stellar-Mass Function (GSMF). A detailed analysis will be presented by Fontana et al. (2003). The present analysis suggests that the evolution of the GSMF and of the stellar mass density is slow with redshift up to $z \simeq 1.5$, in contrast with the rapid evolution of the Galaxy Stellar Mass Function expected in the hierarchical models at $z<2$ (cf. Baugh et al. 2002). In our sample, using galaxy spectral models

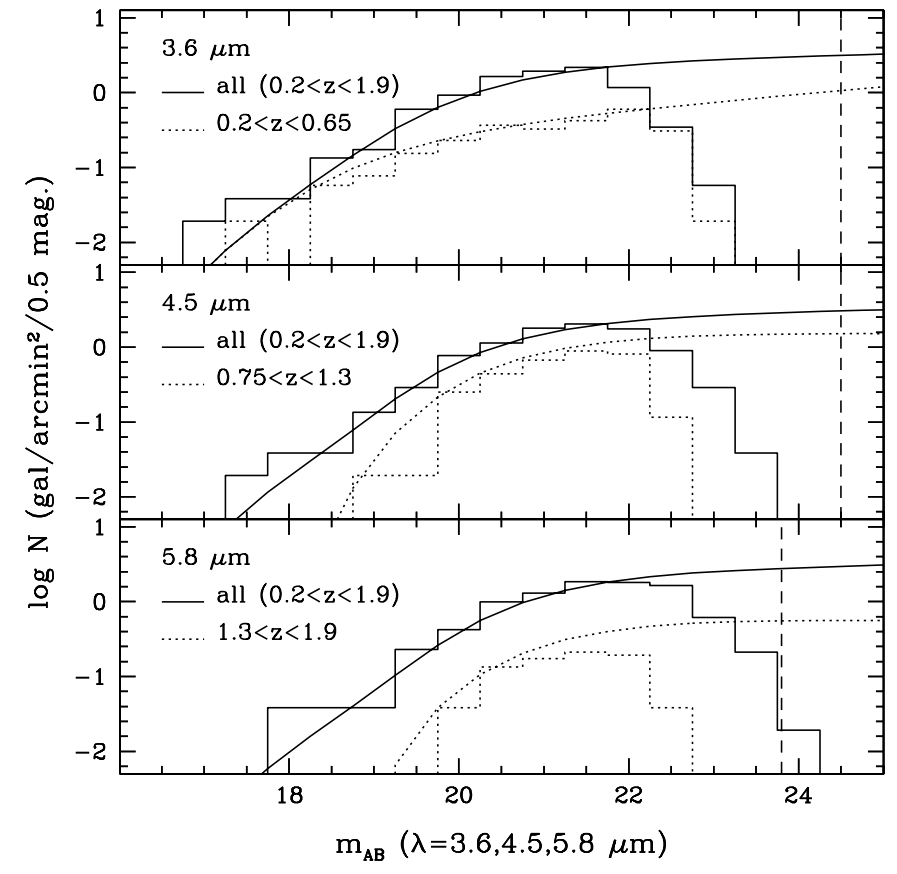

Fig. 13. Predicted SIRTF+IRAC number densities for galaxies with $z<2$ derived from K20 sample (see text) as a function of AB magnitudes at 3 different wavelengths $(\lambda=3.6,4.5,5.8 \mu \mathrm{m})$. Histograms refer to $K_{\mathrm{s}}<20$ galaxies in our sample in the indicated redshift ranges, while lines have been derived from K20 LFs extended to fainter luminosities (using the flat slope in the highest $z$ range). Vertical dashed lines refer to GOODS flux limits at the different wavelengths.

which match colors and spectral types, we found that $\Omega_{\text {stars }}$ decreases by a factor about $1.8 \pm 0.4$ from $z=0$ to $z \simeq 1$, mainly due to the decrease in the mass-to-light ratio, which is however slower than in HMMs models. In at least one of these models (GIF) the small adopted $\mathrm{M} / \mathrm{L}$ ratio corresponds to a color distribution of galaxies at $z \sim 1$ clearly inconsistent with data (see Fig. 12).

Moreover, the fact that the bright-end of the Luminosity Function is dominated by red/early type galaxies and that the mean spectra of early type EROs at $z \simeq 1$ are consistent with an old stellar population (see Paper I), suggest that old and massive elliptical galaxies were already in place at $z \simeq 1$ (see also Paper I) and, therefore, they should have formed their stars and assembled their mass at higher redshift. These results are in contrast with the current renditions of hierarchical models in which the bulk of massive elliptical galaxies forms through merging at low redshift $(z<1-2)$ and suggest that most of the merging which form elliptical galaxies in the hierarchical models should occur at higher redshift (say, $z>2-3$ ), followed by a pure "luminosity-like" evolution. On the other hand, HMMs overpredict the density of low-luminosity, low mass, galaxies at $z<1$, as previously noted in the local universe (cf. Fig. 1 in Baugh et al. 2002) and in the $B$ and UV rest frame bands at $z \geq 1$ (Poli et al. 2001; Somerville et al. 2001; Poli et al. 2003).

Our results show the importance of sampling the faintend of the LF in even deeper near-IR selected samples. In addition, to derive the LF at $z \geq 1$ in a wider but shallow sample (say $K<18.5$ ) will be essential to trace the evolution of the most massive galaxies with higher statistical significance. 
In the near future direct measurements of rest-frame $K$-band magnitude for high redshift galaxies will be possible with space-based infrared surveys, in particular the SIRTF (Space Infrared Telescope Facility) Legacy Science project GOODS (Great Observatories Origins Deep Survey, P.I. M. Dickinson). Our data allow to directly estimate the number densities of $z<2$ galaxies at the different SIRTF-GOODS wavelengths, $\lambda=3.6,4.5,5.8 \mu \mathrm{m}$, which sample the rest-frame $K_{\mathrm{s}}$-band at $z \simeq 0.7,1.1,1.7$ respectively, similar to the $z_{\text {mean }}$ adopted for the K20 LFs. We have converted the absolute $M_{K_{\mathrm{s}}}$ magnitudes, derived here for the K20 sample, to SIRTF+IRAC fluxes using the galaxy spectral models described in Sect. 3 for normal galaxies and the spectral model for M 82 (Silva et al. 1998) for the dusty star forming galaxies. The expected number densities for objects with $z<2$ are shown in Fig. 13. At the expected depth of GOODS (see Dickinson et al. 2002b), SIRTF will detect about 5000 galaxies at $z<2$ within $330 \operatorname{arcmin}^{2}$. GOODS SIRTF Legacy Program, sampling the rest frame near-IR luminosities directly, will allow to derive the $K$-band Luminosity Function along with the stellar mass distribution well below the K20 limit (see Fig. 13) and with higher statistical significance. With these data the stellar mass assembly history of galaxies will be measured over a wide range of redshifts and cosmic time.

We make publicy available the $1 / V_{\max }$ estimates at http://www.arcetri.astro.it/ k20/releases/index.html

Acknowledgements. Part of this work was supported by the MURST (Cofin 2000-2001), ASI and by the European Community Research and Training Network "The physics of the intergalactic medium". We are in debt with Carlton Baugh for providing the HMM predictions.

\section{References}

Barger, A. J., Cowie, L. L., \& Sanders, D. B. 1999, ApJ, 518, L5

Baugh, C. M., Benson, A. J., Cole, S., Frenk, C. S., \& Lacey, C. 2002, in The Mass of Galaxies at Low and High Redshift, ESO/USM Workshop, Venice, Italy, ed. R. Bender, \& A. Renzini (SpringerVerlag), 91 [astro-ph/0203051]

Bolzonella, M., Miralles, J.-M., \& Pelló, R. 2000, A\&A, 363, 476

Boselli, A., Gavazzi, G., Donas, J., \& Scodeggio, M. 2001, AJ, 121, 753

Broadhurst, T., Ellis, R. S., \& Grazebrook, K. 1992, Nature, 355, 55

Broadhurst, T., \& Bouwens, R. J. 2000, ApJ, 530, L53

Bruzual, G., \& Charlot, S. 1993, 405, 538

Cimatti, A., Daddi, E., Mignoli, M., et al. 2002a, A\&A, 381, L68 (Paper I)

Cimatti, A., Mignoli, M., Daddi, E., et al. 2002b, A\&A, 392, 395 (Paper III)

Cimatti, A., Pozzetti, L., Mignoli, M., et al. 2002c, A\&A, 391, L1 (Paper IV)

Cohen, J. G. 2002, ApJ, 567, 672

Cohen, J. G., Blandford, R., Hogg, D. W., et al. 1999, ApJ, 512, 30

Cole, S., Lacey, C. G., Baugh, C. M., \& Frenk, C. S. 2000, MNRAS, 319, 168

Cole, S., Norberg, P., Baugh, C. M., et al. 2001, MNRAS, 326, 255

Cowie, L. L., Gardner, J. P., Hu, E. M., et al. 1994, ApJ, 434, 114

Cowie, L. L., Songaila A., Hu, E. M., \& Cohen, J. G. 1996, AJ, 112, 839

Cowie, L. L., Songaila, A., \& Barger, A. J. 1999, AJ, 118, 603
Daddi, E., Cimatti, A., Broadhurst, T., et al. 2002, A\&A, 384, L1 (Paper II)

Dickinson, M., Papovich, C., Ferguson, H. C., \& Budavari, T. 2002a, ApJ, in press [astro-ph/0212242]

Dickinson, M., Giavalisco, M., \& the GOODS team 2002b, in The Mass of Galaxies at Low and High Redshift, ESO/USM Workshop, Venice, Italy, ed. R. Bender, \& A. Renzini (SpringerVerlag), 324 [astro-ph/0204213]

Feulner, G., Bender, R., Drory, N., et al. 2003, MNRAS, in press

Firth, A. E., Somerville, R. S., McMahon, R. G., et al. 2002, MNRAS, 332,617

Fontana, A., D’Odorico, S., Poli, F., et al. 2000, AJ, 120, 2206

Fontana, A., et al. 2003, in preparation

Gavazzi, G., Pierini, D., \& Boselli, A. 1996, A\&A, 312, 397

Giacconi, R., Rosati, P., Tozzi, P., et al. 2001, ApJ, 551, 624

Hughes, D. H., Serjeant, S., Dunlop, J., et al. 1998, Nature, 394, 24

Im, M., Faber, S. M., Gebhardt, K., et al. 2001, AJ, 122, 750

Im, M., Simard, L., Faber, S. M., et al. 2002, ApJ, 571, 136

Jarrett, T. H., Chester, T., Cutri, R., et al. 2000, AJ, 119, 2498

Kauffmann, G., Colberg, J. M., Diaferio, A., \& White, S. D. M. 1999, MNRAS, 303, 188

Kauffmann, G., Heckman, T. M., White, S. D. M., et al. 2002, MNRAS, in press [astro-ph/0205070]

Kennicutt, R. C. Jr. 1983, ApJ, 272, 54

Kochanek, C. S., Pahre, M. A., Falco, E. E., et al. 2001, ApJ, 560, 566

Lilly, S. J., Tresse, L., Hammer, F., Crampton, D., \& Le Fevre, O. 1995, ApJ, 455, 108

Lilly, S. J., Le Fevre, O., Hammer, F., \& Crampton, D. 1996, ApJ, 460, L1

Madau, P., Ferguson, H. C., Dickinson, M. E., et al. 1996, ApJ, 283, 1388

Madau, P., Pozzetti, L., \& Dickinson, M. 1998, ApJ, 498, 106

Mannucci, F., Basile, F., Poggianti, B. M., et al. 2001, MNRAS, 326, 745

Menanteau, F., Abraham, R. G., \& Ellis, R. S. 2001, MNRAS, 322, 1

Menci, N., Cavaliere, A., Fontana, A., Giallongo, E., \& Poli, F. 2002, ApJ, 575, 18

Poli, F., Menci, N., Giallongo, E., et al. 2001, ApJ, 551, L45

Poli, F., et al. 2003, in preparation

Pozzetti, L., Bruzual, A. G., \& Zamorani, G. 1996, MNRAS, 281, 953

Pozzetti, L., Madau, P., Zamorani, G., Ferguson, H. C., \& Bruzual, A. G. 1998, MNRAS, 298, 1133

Pozzetti, L., \& Madau, P. 2001, in The Extragalactic Infrared Background and its Cosmological Implications, International Astronomical Union. Symp. No. 204. Manchester, England, August 2000, ed. M. Harwit, \& M. G. Hauseri, p. 71 [astro-ph/0011359]

Salpeter, E. E. 1955, ApJ, 121, 161

Sandage, A., Tammann, G. A., \& Yahil, A. 1979, ApJ, 232, 352

Scalo, J. M. 1986, Fundam. Cosm. Phys., 11, 1

Schmidt, M. 1968, ApJ, 151, 393

Schechter, P. 1976, ApJ, 203, 297

Silva, L., Granato, G. L., Bressan, A., \& Danese, L. 1998, ApJ, 509, 103

Smith, G. P., Smail, I., Kneib, J. P., et al. 2002, MNRAS, 330, 1

Steidel, C. C., Adelberger, K. L., Giavalisco, M., Dickinson, M., \& Pettini, M. 1999, ApJ, 519, 1

Somerville, R. S., Primack, J. R., \& Faber, S. M. 2001, MNRAS, 320, 504

Totani, T., Yoshii, Y., Maihara, T., Iwamuro, F., \& Motohara, K. 2001, ApJ, 559, 592

Zucca, E., Pozzetti, L., \& Zamorani, G. 1994, MNRAS, 269, 953

Zucca, E., Zamorani, G., Vettolani, G., et al. 1997, A\&A, 326, 477 